\title{
The unusual journey of a retained epicardial pacing wire
}

\author{
Francesca Giacomazzi, ${ }^{1}$ Lorenzo Menicanti, ${ }^{2}$ Massimo Lombardi, ${ }^{3}$ Roberto Tramarin ${ }^{1}$ \\ ${ }^{1}$ Cardiac Rehabilitation Unit; ${ }^{2}$ Cardiac Surgery Department; ${ }^{3}$ Multimodal Cardiac Imaging, IRCCS Policlinico San Donato, \\ Milano, Italy
}

\begin{abstract}
The placement of temporary epicardial pacing wires (EPWs) at the completion of cardiac surgery is a routine procedure in most centers. Complications related to their insertion, removal and retention are infrequent, yet potentially severe, including hemorrhage, tamponade, infection and death. Here, we describe an unusual case of retained temporary EPWs migration.
\end{abstract}

\section{Case Report}

A 68-year-old Caucasian man was admitted to our Institute for worsening heart failure. His medical history included arterial hypertension, dyslipidemia, chronic obstructive pulmonary disease and hypothyroidism. The patient had an anterior ST-elevation myocardial infarction and underwent a primary percutaneous coronary intervention + drug-eluting stents on proximal left anterior descending artery. Subsequently he showed an unfavorable left ventricular (LV) remodeling leading to a dilated cardiomyopathy with apical aneurism, reduced left ventricle systolic function (28\%), functional severe mitral and tricuspid regurgitation. Six months later, the patient underwent double coronary artery bypass grafting, mitral annuloplasty, tricuspid annuloplasty with Carpentier's annulus and left ventricular surgical restoration. During open-heart surgery two epicardial wires [Myopace; FIAB Spa, Vicchio (FI), Italy] were applied on the right atrium and right ventricle respectively, for postoperative rescue external pacing (Figure 1). Due to an

Correspondence: Francesca Giacomazzi, Cardiac Rehabilitation Unit, IRCCS Policlinico San Donato, Piazza Edmondo Malan 2, 20097 San Donato Milanese (MI), Italy.

E-mail: francesca.giacomazzi@grupposandonato.it

Key words: Temporary epicardial pacing wire migration; epicardial pacing wire complication.

Conflict of interest: the authors declare no potential conflict of interest.

Received for publication: 11 July 2017.

Accepted for publication: 7 August 2017.

This work is licensed under a Creative Commons Attribution NonCommercial 4.0 License (CC BY-NC 4.0).

(C) Copyright F. Giacomazzi et al., 2017

Licensee PAGEPress, Italy

Italian Journal of Medicine 2017; 11:396-398

doi:10.4081/itjm.2017.905 abnormal extraction resistance, one of the cables could not be removed; therefore, after a slight tension, it was cut at the skin level and left in place.

The patient was discharged in stable clinical conditions, in Class II of the New York Heart Association (NYHA) classification, with improved LV function (ejection fraction $40 \%$ ).

Ten months later, he was readmitted to our hospital for worsening heart failure. At admission, the patient was in NYHA Class III, blood pressure 105/65 mmHg, heart rate $74 \mathrm{bpm}$, oxygen saturation $96 \%$ in room air. On physical examination important jugular vein distention, bibasilar rales, hepatomegaly and ankle edema were present; S3 sound was also evident. On lab test anemia (hemoglobin $11 \mathrm{~g} / \mathrm{dL}$ ), elevated NT-Pro-BNP $(9000 \mathrm{ng} / \mathrm{mL})$, cholestasis and hyponatremia (130 $\mathrm{mEq} / \mathrm{L}$ ) were present.

Electrocardiogram showed RSR at $74 \mathrm{bpm}, 1^{\text {st }}$ degree atrioventricular bloc and right bundle branch block. Congestion of small circulation was evident on chest X-ray.

To assess postoperative left ventricular long-term remodeling, the patient underwent cardiac magnetic resonance imaging (MRI) with gadolinium, which confirmed severe biventricular systolic dysfunction with anterior scar and paradoxical motion of interventricular septum. Two days later, the patient sought medical attention because of the appearance of a foreign body at the skin level of the abdomen, $5 \mathrm{~cm}$ above the umbilicus. By gently pulling it, a $10-\mathrm{cm}$-long epicardial wire was removed. After medical therapy for heart failure, the patient was discharged and referred to our Heart Failure Outpatient Unit. Two months later, he successfully underwent implantation of implantable cardioverter defibrillator-cardiac resynchronization therapy.

\section{Discussion}

Epicardial pacing wires (EPWs) are intended for mono- or bicameral temporary cardiac electrostimu- 
lation via the epimyocardium by connection with external cardiac pacemakers. They are sutured to the right atrium and the right ventricle during the final phase of cardiac surgery to ensure temporary pacing when needed. Their use is not recommended in case of implanted pacemakers, for cardioversion or for permanent electrostimulation. EPWs consist of stainless multithread conductors with an external insulating sheath. One end of the conductor is connected with a cardiac needle and is exposed in order to create a stimulation surface area typically of $9.4 \mathrm{~mm}^{2}$ in the adult versions. The myocardial electrodes are fixed to the epimyocardial tissue in order to ensure both an effective attachment and a low risk of traction damage during its removal. At the other end, the conductor is crimped into the eye of a needle, which is plugged into the external pacemaker. Binding of the electrodes on the myocardium must be loose.

In most EPW brands, the conductors must adhere

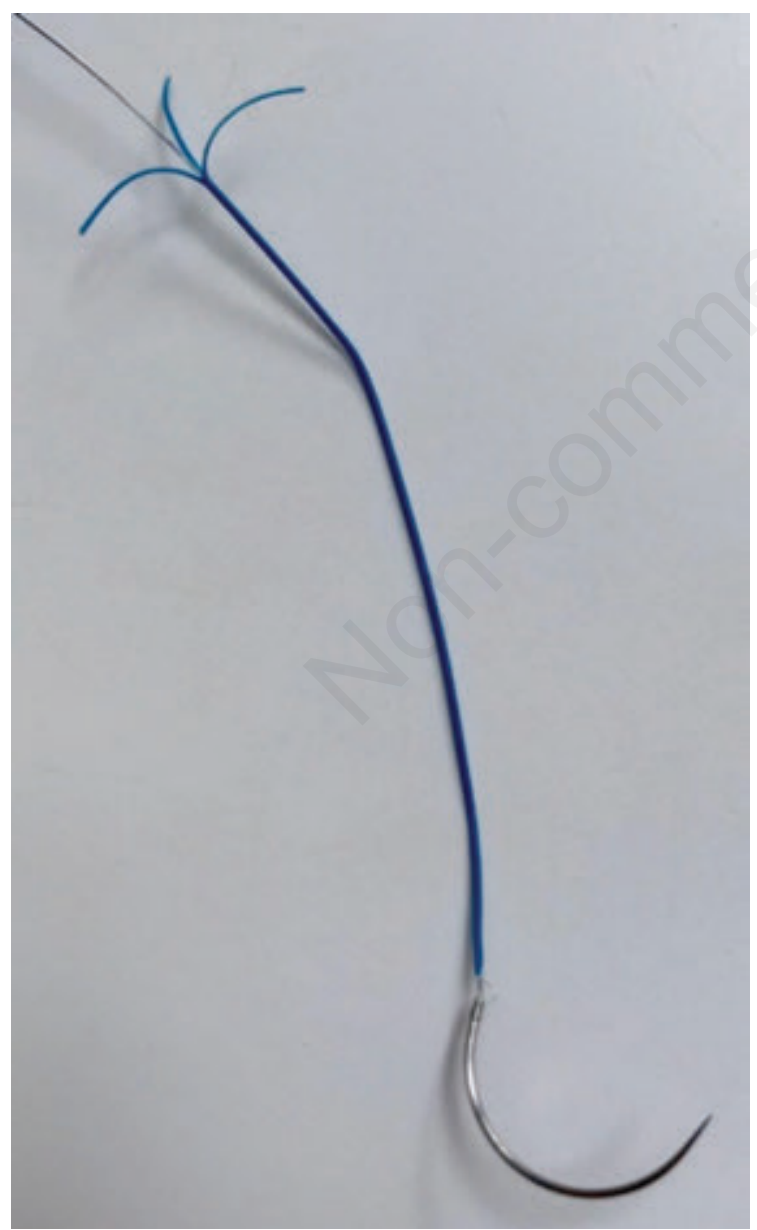

Figure 1. Myopace (FIAB Spa) epimyocardial electrodes are usually applied on the right atrium and right ventricle at the end of cardiac surgery, for postoperative rescue external pacing. to the myocardium through a suture. Wires stability can be ensured by a single stich in the outlet point of the thoracic wall to avoid the dislocation of the electrode by accidental tractions. Conventionally, the ventricular EPW is exteriorized left and the atrial one right. Usually for EPW removal a light traction allows the complete extraction of the wire. To prevent tissue reaction producing bridles interfering with EPW removal, extraction should be carried out as soon as the electrostimulation is no longer required and, in any case, within 20 days from implantation.

In most cases EPWs are removed before discharge from postoperative Intensive Care Unit; occasionally they are cut just under the skin level in case of thrombocytopenia or of high risk of bleeding. On other occasions, the unsuccessful attempt of removal with a gentle traction, force their cut to avoid the risk of significant injury to the right atrium or ventricle.

With wider diffusion and higher level of recommendation on magnetic resonance application as goal standard for different diseases, an increasing number of patients is undergoing this technique.

The stainless guide inside represents a metallic foreign body, potentially causing local and distant complications, most of which occurring in the chest, less frequently in the abdomen or in the jaw.

Reported complications related to retained EPW including valvular endocarditis ${ }^{1,2}$ ventricular tachycardia, ${ }^{3}$ migration into the lung, ${ }^{4}$ into the colon ${ }^{5}$ and late development of posterior mediastinal abscess ${ }^{6}$ are rare but potentially severe. Although rare, a few other cases of mediastinal abscess in association with retained EPW have been reported. In one case, an EPW was found inside an anterior mediastinal abscess cavity 12 years after coronary artery bypass surgery. ${ }^{7}$ In another case atrial temporary EPW migrated into the aortic arch via the artificial graft while ventricular EPW moved into the right pulmonary artery via right ventricle one year after emergent aortic hemiarch graft replacement with open stent graft implantation into the distal aortic arch for acute type A aortic dissection. ${ }^{8}$ Rarely, bleeding inside the pericardium with tamponade occurs after EPW removal.

Heating of stainless wires represents the major problem determining MRI safety. Although there is a theoretical risk that MRI examination in patients with retained temporary epicardial wires - which consist of electrically conductive materials - could lead to cardiac excitation or thermal injury, being EPWs relatively short in length, they do not usually form large loops, and are not believed to pose a significant hazard during MRI procedures. ${ }^{9}$ In fact, many patients with temporary EPWs have undergone MRI scanning without acute complications, even if this is not believed to represent a safe procedure and warning about not to subject to MRI patients with implanted Myopace 
(FIAB Spa) is well described in the instruction for use of epimyocardial electrodes' manual. ${ }^{10}$

Here, we describe a case of retained EPW's migration to the abdominal skin after MRI execution. In order to test the hypothesis whether the EPW's migration could be ascribed to the recent immersion in a magnetic field, an epimyocardial electrode of the same brand (Myopace; FIAB Spa) was placed near MRI machinery for few seconds, with no effect.

It could not be excluded, though, that heating of the retained wires during the exam had favored its movement and subsequent migration to the skin.

Even if the presence of a retained EPW does not represent a real contraindication to MRI exam at the moment, no information about potential MRI effect on facilitating migration of retained EPWs is available, due to lack of retrospective and prospective studies.

Therefore, in case of retained EPW it is important for doctors, patients and MRI technicians to be aware of the presence of a stainless foreign body. Furthermore, surgeons in charge of extracting EPWs should remember to alert the patient about the presence of a foreign body when left in place.

\section{Conclusions}

The retention of EPWs after cardiac surgery may cause a wide range of medical and surgical complications, and although they are rare they occur with severe morbidity. Even though these cases should not be considered completely fully safe for potential wires heating, many patients have undergone the exam without evident complications. Nevertheless, lack of any data on the potential long-term MRI effect on retained temporary EPWs should promote retrospective analysis and prospective studies.

\section{References}

1. Mansur AJ, Grinberg M, Costa R, et al. Dura mater valve endocarditis related to retained fragment of postoperative temporary epicardial pacemaker lead. Am Heart J 1984;108:1049-52.

2. Dyal HK, Sehgal R. The catastrophic journey of a retained temporary epicardial pacemaker wire leading to Enterococcus faecalis endocarditis and subsequent stroke. BMJ Case Rep 2015 [Epub ahead of print].

3. Meier DJ, Tamirisa KP, Eitzman DT. Ventricular tachycardia associated with transmyocardial migration of an epicardial pacing wire. Ann Thorac Surg 2004;77: 1077-9.

4. Horng GS, Ashley E, Balsam L, et al. Progressive dyspnea after $\mathrm{CABG}$ : complication of retained pacing wires. Ann Thorac Surg 2008;86:1352-4.

5. Gonzales S, White H, Echavarria J. Transcolonic migration of retained epicardial pacing wires. Case Rep Radiol 2015;2015:416587.

6. Smith DE 3rd, DeAnda A Jr, Towe CW, Balsam LB. Retroaortic abscess: an unusual complication of a retained epicardial pacing wire. Interact Cardiovasc Thorac Surg 2013;16:221-3.

7. Domaradzki W, Olszowka P, Mrozek R, et al. Mediastinal abscess as a remote complication of CABG combined with temporary cardiac pacing-a case report. Kardiol Pol 2005;63:70-2.

8. Domoto S, Nakazawa K, Tabata M, Niinami H. Migration of retained epicardial pacing wires. Eur Heart J 2016;37:3246.

9. Baikoussis NG, Apostolakis E, Papakonstantinou NA, et al. Safety of magnetic resonance imaging in patients with implanted cardiac prostheses and metallic cardiovascular electronic devices. Ann Thorac Surg 2011;91: 2006-11.

10. FIAB Spa. Myopace epimyocardial electrodes and Myoconnector plug - Instructions for use; available from: http://www.fiab.it/en/prodotti.php?id=109 